\title{
Gentechnisch veränderte Tiere
}

Bereits in den 80er Jahren konnte ein fremder DNA-Abschnitt in das Erbgut einer Maus eingebaut werden. Kurze Zeit später eilten Meldungen über die ersten transgenen Nagetiere um die Welt. Doch in Summe ist die gentechnische Veränderung von Tieren, vor allem bei Säugetieren, im Vergleich zu Mikroorganismen oder Pflanzen bedeutend schwieriger. Bei Tieren genügt es nicht, eine Körperzelle in der Zellkultur zu verändern, denn aus einer isolierten Kuhzelle wird keine Kuh. Es müssen Eizellen oder befruchtete Eizellen manipuliert werden und zumeist künstliche Befruchtungen vorgenommen werden. Viele Projekte mit veränderten Schweinen, Schafen oder Kühen endeten unter anderem aufgrund der größeren Herausforderung als Flop, weil die Tiere krankheitsanfällig waren oder unter Organ- und Gelenkschäden litten. Viele Entwicklungen verliefen sich auch aus Kostengründen im Sand. Gentechnisch veränderte Säugetiere werden daher derzeit ausschließlich für die Medikamentenproduktion eingesetzt. Deutlich einfacher ist die genetische Manipulation etwa bei Fischen oder Insekten. Somit sind es derzeit auch ebendiese Tiergruppen, die als gentechnisch veränderte Tiere bereits eine Zulassung haben bzw. in absehbarer Zeit bekommen werden. Die neuen Verfahren des Genome Editing zur Veränderung der DNA werden den gesamten Bereich der Gentechnik revolutionieren und auch der gentechnischen Veränderung von Tieren ganz neue Möglichkeiten eröffnen. An dieser Stelle sollen anhand von Beispielen Theorie und Praxis transgener Tiere beleuchtet werden.

\subsection{Fische}

Die gentechnisch veränderten, schneller wachsenden („Turbo“-) Lachse aus den USA, sind bereits seit Jahren in den Schlagzeilen um die Markteinführung. Dass die Forschung bei Fischen weit fortgeschritten ist, liegt an der einfachen Hand-

(C) Springer Fachmedien Wiesbaden 2017

O. Luger, Gentechnik geht uns alle an!,

DOI 10.1007/978-3-658-15605-3_5 
habung, da eine große Eimenge zur Verfügung steht und sowohl Befruchtung als auch Entwicklung außerhalb des Körpers stattfinden. Das ist wohl auch der Grund, warum viele verschiedene Fischarten in mehreren Ländern (z.B.: USA, Kanada und Japan) gentechnisch verändert werden. Die Veränderungen gehen vor allem in Richtung schnelleres Wachstum bzw. Steigerung der Muskelmasse. Daneben wird aber auch an Anpassungen an andere Wassertemperaturen und an Krankheitserreger geforscht. Neben den gentechnisch veränderten Lachsen haben auch die veränderten Zierfische unter dem Namen Glofish eine Zulassung.

Die Turbolachse (Handelsname AquAdvantage) der Firma AquaBounty Technologies sind die ersten transgenen Tiere, die für den Verzehr durch den Menschen gedacht sind. Dabei wurden dem atlantischen Lachs ein Gen für ein Wachstumshormon des Königslachses und ein Regulationsgen eingesetzt, die gemeinsam zu einem bedeutend schnelleren Wachstum führen. Die gentechnisch veränderten Lachse erreichen - verglichen mit nicht veränderten atlantischen Lachsen - bereits in der halben Zeit (1,5 Jahre) ihr Schlachtgewicht (siehe Abb. 5.1). Angesichts der schwindenden Fischbestände und der großen Nachfrage ist dies natürlich ein interessantes Geschäftsfeld. Die Fischeier für die Aquakulturen werden in Kanada produziert und die Aufzucht der Fische erfolgt in Panama. Der Widerstand in den USA, wo die Zulassung zum Verzehr schon vor Jahren beantragt wurde, ist allerdings groß. 2014 haben viele große Handelsketten bekannt gegeben, den gentechnisch veränderten Lachs nicht zu verkaufen, sollte er zugelassen werden. Die zuständige Zulassungsbehörde in den USA hat die Lachse bereits 2010 nach den Richtlinien zur Bewertung von Lebensmitteln von gentechnisch veränderten Tieren als unbedenklich eingestuft. Die Zulassung verzögerte sich aber immer wieder bis Ende 2015. Seither darf der Lachs aber ohne besondere Kennzeichnung in den USA verkauft werden. Die Zulassungsbehörde hat eine Kennzeichnung lediglich empfohlen.

\footnotetext{
Abb. 5.1

Gentechnisch veränderter Lachs: Die Abbildung zeigt einen gentechnisch veränderten Lachs (Hintergrund) und einen natürlichen Lachs (Vordergrund) im Vergleich. (c) AquaBounty Technologies
}

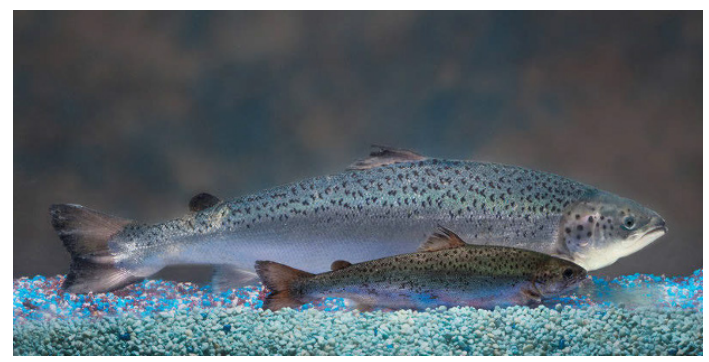




\section{Infobox}

Aquakulturen sind abgegrenzte Zonen in natürlichen Gewässern, abgegrenzte künstliche Teiche oder geschlossene Gefäße zur Aufzucht von Wasserlebewesen (Fische, Muscheln, Garnelen, ...).

Hormone sind Botenstoffe des Körpers, die von Organen bzw. Geweben oder Drüsen gebildet werden und auf bestimmte Zielzellen wirken. Das Hormonsystem ist ein sensibler Steuerungsmechanismus. Ein Beispiel hierfür ist Insulin (siehe Kapitel 7.1).

In der Praxis zeigt sich, dass Umweltbehörden und Fischereiverbände die Zulassung vehement ablehnen. Ein wichtiges Argument dabei ist, dass die veränderten Lachse aus den Tanks der Aquakulturen entkommen könnten, sich verbreiten und andere Fischarten verdrängen. Um dem gegenzusteuern, sollen nur unfruchtbare Weibchen vermarktet werden und die Tanks der Aquakulturen nicht an natürlichen Gewässern gelegen sein. Letztlich kann aber ein Entkommen transgener Tiere nie hundertprozentig ausgeschlossen werden. Die Folgen sind völlig unabsehbar und nicht rückgängig zu machen.

Betrachtet man die Probleme der normalen Lachs-Aquakulturen in Norwegen oder anderswo, so ist fraglich, ob man diese Lachse - gleichgültig, ob gentechnisch verändert oder nicht - überhaupt essen mag. Die Belastung der Fische mit Medikamenten und anderen Stoffen ist enorm und von gesundem Fisch kann wohl kaum noch die Rede sein. Auch die Belastung der Gewässer durch diese Art der Fischhaltung ist beträchtlich.

Durch die erhöhte Menge an Wachstumshormonen in den veränderten Tieren ist fraglich, welche Auswirkungen diese Extrahormondosis auf den Konsumenten bzw. die Konsumentin hat. Außerdem kann man die Fleischqualität anzweifeln, wenn die Tiere in derart kurzer Zeit heranwachsen. In den nächsten Jahren wird sich zeigen, wie die Konsumentinnen und Konsumenten auf den neuen Lachs reagieren. Offen ist derzeit noch, wie die Handelsketten die freiwillige Kennzeichnung handhaben und ob die Konsumentinnen und Konsumenten eine Wahl haben werden.

Bereits im Handel erhältlich sind gentechnisch veränderte Zierfische. Unter dem Handelsnamen Glofish sind bereits drei Fischarten in verschiedenen Farben erhältlich. Den Tieren wurde ein Gen für ein fluoreszierendes Protein je nach Farbe zum Beispiel von einer Koralle oder einer Qualle eingebaut. Damit entstanden 2004 die ersten gentechnisch veränderten Haustiere. In der Europäischen Union ist der Vertrieb und die Zucht von gentechnisch veränderten Tieren verboten, aber es hat natürlich nicht lange gedauert, bis die ersten illegal eingeführten Tiere in 
Deutschland aufgetaucht sind. In den USA ist für den Handel mit Glofish nicht einmal eine Genehmigung erforderlich. Die Folgen von entkommenen gentechnisch veränderten Fischen in die freie Wildbahn werden zwar in den US-Medien diskutiert, aber die Behörden sind bisher untätig, weil es sich um kein Lebensmittel handelt.

\subsection{Insekten}

Insekten sind die artenreichste Tierklasse, haben nahezu alle Lebensräume besiedelt und spielen verschiedenste ökologische Rollen, die großteils als unentbehrlich bezeichnet werden können. Neben den beliebten Nützlingen, wie der Honigbiene, wäre die Liste an, für den Menschen oder das Ökosystem, vorteilhaften Insekten wohl schier unendlich.

Für den Menschen haben aber manche Insekten die ungünstige Eigenschaft, Krankheitserreger zu übertragen oder Nutzpflanzen zu essen. Vor allem zum zweiten Punkt muss bemerkt werden, dass die technisierte Landwirtschaft mit den Monokulturen ein massenhaftes Vermehren der Schädlinge erst ermöglicht. Die gentechnische Entwicklung der letzten Jahre hat veränderte Insekten hervorgebracht, die die Fortpflanzung der jeweiligen Art beeinträchtigen soll. Somit dezimiert man durch den Eingriff ganze Insektenarten bzw. rottet diese gezielt aus. Diese Technologie wurde bereits in den 1950er Jahren mit Hilfe radioaktiv bestrahlter und somit sterilisierter Insekten in den USA entwickelt und erfolgreich eingesetzt. Die radioaktive Bestrahlung kann mittlerweile durch gentechnische Eingriffe ersetzt werden. Die eingebauten Gene in das Erbgut männlicher Insekten stören die Zellfunktionen beim Nachwuchs und führen so zu deren Absterben (Release of Insects carrying a Dominant Lethal kurz RIDL - Technik) ${ }^{[42]}$ Bei der Tigermücke oder der Olivenfliege aber auch anderen Insektenarten wurde diese Technik bereits angewandt.

\section{Infobox}

Bei der "Release of Insects carrying a Dominant Lethal“ kurz „RIDL - Technik“ handelt es sich um eine bereits 2000 beschriebene Vorgehensweise. Wie der Name schon sagt, handelt es sich um eine Technik der Insektenbekämpfung, indem manipulierte Tiere mit einem dominaten, tödlichen Gen freigelassen werden. Dieses Gen, dabei gibt es verschiedene Möglichkeiten, beeinträchtigt dann die Fortpflanzung und verhindert fruchtbare Nachkommen. 
Gene Drive ist eine Technik, bei der sich das gentechnisch veränderte Gen immer durchsetzt und sogar die zweite Variante des Gens umschreibt. Somit erhalten unabhängig von den Merkmalen der Geschlechtspartner nahezu 100 Prozent der Nachkommen das veränderte Gen und dadurch das entsprechende Merkmal. Innerhalb weniger Generationen können so ganze Populationen unumkehrbar verändert werden.

Diese Technik wurde zum Beispiel von Oxitec (das britische Unternehmen wurde im Sommer 2015 von Intrexon, einem US-amerikanischen Unternehmen übernommen) bei der ägyptischen Tigermücke eingesetzt. Diese Mückenart überträgt die Erreger des Dengue-Fiebers und Gelbfiebers. In verschiedenen Freisetzungsversuchen konnten die örtlichen Populationen um 80 und mehr Prozent dezimiert werden. 2014 bekam diese gentechnisch veränderte Tigermücke in Brasilien die Zulassung. Die rasche Ausbreitung des Zika-Virus Anfang 2016, das unter anderem auch von dieser Mückenart übertragen wird, gibt dem Kampf gegen die Tigermücke neuen Aufwind. Die gentechnisch veränderten Mücken könnten durch diese neue, zusätzliche Bedrohung auch in anderen, betroffenen Ländern eine Zulassung erhalten. Ähnliche Techniken werden auch zur Bekämpfung der Malaria übertragenden Mücken bereits getestet, wie eine Studie von Anfang 2016 zeigt. ${ }^{[43]}$

Viele Umwelt- und Landwirtschaftsorganisationen laufen wegen der vielen Risiken Sturm gegen die Zulassung von gentechnisch veränderten Insekten. Sie warnen besonders vor Organismen mit einem so genannten "Gene Drive“ Effekt. Unter "Gene Drive“ versteht man den Einbau von Genen, die sich auf die Häufigkeit der Vererbung auswirken. Während Merkmale normalerweise nur an einen Teil der Nachkommen weitergegeben werden, gibt es gentechnische Verfahren, die zu einer Weitergabe der gentechnisch veränderten Merkmale auf alle Nachkommen führen. Dieses neue Merkmal würde sich extrem schnell in natürlichen Populationen verbreiten, weil es sich allen Wildformen aufzwingt (siehe Abb. 5.2). Mit dieser Technik lassen sich Arten unwiederbringlich und vollständig verändern, ohne eine Chance, dass sich das veränderte Merkmal im Laufe der Zeit durch Kreuzungen mit Wildformen ausdünnt. Internationale Regelungen oder Verbote gegen die Verwendung derartiger Technologien gibt es nicht. 


\section{KLASSISCHE VERERBUNG}

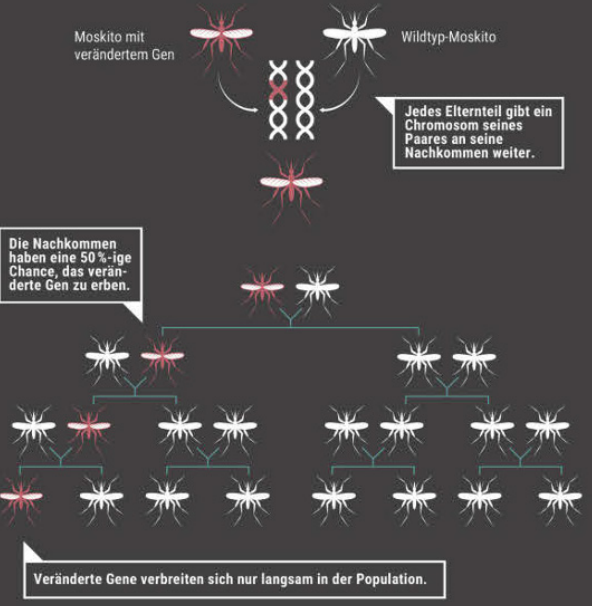

\section{VERERBUNG DURCH GENE DRIVE}

Das Gene-Drive-System schneidet das Partner -Chromosom, und das Reparatursystem der Zelle kopiert die Veränderung des ersten Chromosoms auf das zweite:
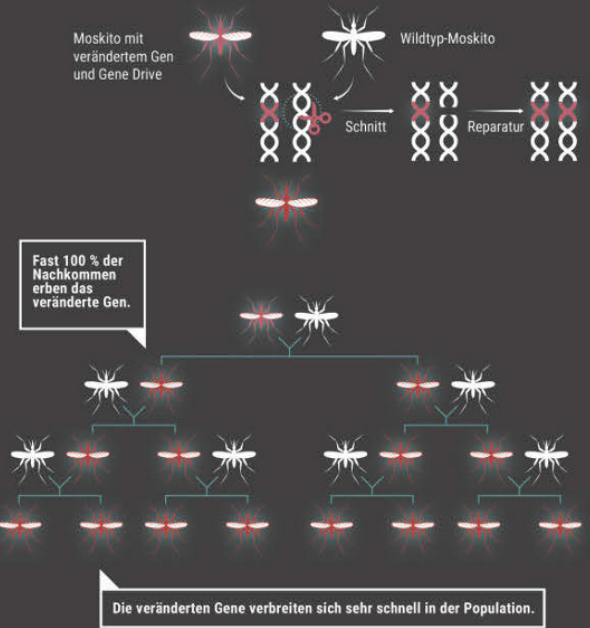

Abb. 5.2 Gene Drive: Diese Gegenüberstellung von klassischer Vererbung und Vererbung durch Gene Drive zeigt die unterschiedliche Häufigkeit der veränderten Gene 
in der Population. Während bei der klassischen Vererbung 50 Prozent der Nachkommen das veränderte Gen tragen und es im Laufe der Zeit zu einer Ausdünnung kommt, sind bei der Vererbung durch Gene Drive (nahezu) 100 Prozent der Nachkommen betroffen. In einigen Generationen kann so die gesamte Population verändert werden. (c) Wesley Fernandes/Nature; dt. Bearbeitung: Spektrum der Wissenschaft; Ledford, H.: CRISPR, the disruptor. In: Nature 522, S. 20-24, 2015 (Ausschnitt)

Die Bevölkerung der Regionen, in denen Dengue - Fieber eine verbreitete Krankheit ist, nimmt das Risiko der gentechnischen Veränderung natürlich gerne in Kauf, weil die Bedrohung durch die Krankheit um ein Vielfaches beängstigender ist. Aus der Entfernung betrachtet ist die Entwicklung allerdings sehr rasch vorangeschritten und basiert auf einer einzigen Technologie, nämlich der RIDL - Technik der Firma Oxitec. Gerade Insekten sind bekannt dafür, schnell Resistenzen auszubilden, was auch bei dieser Technik möglich ist. Wenn die gentechnisch veränderten Mücken also nicht, wie vorgesehen, absterben, ist nicht absehbar, wie sie sich in freier Wildbahn weiterentwickeln und verhalten werden. Ähnlich wie schon bei den Bt-Giften beschrieben, kann der Erfolg der Methode zu einer Ausrottung des Hauptschädlings führen, was die ökologische Nische für andere Insekten öffnet. Es wird somit das Problem möglicherweise nur kurzfristig beseitigt werden.

Eine Art gezielt auszurotten, hat immer Folgen für das ökologische Zusammenspiel. Keine Art ist isoliert, sondern über vielfältigste Wechselbeziehungen (Nahrungsbeziehungen, Symbiosen, ...) mit anderen verbunden. Die Folgen einer Ausrottung oder extremen Dezimierung können verschiedenste Auswirkungen auch auf andere Lebewesen oder Lebensräume haben, deren Zusammenhänge vielleicht nicht bekannt oder nicht gut genug erforscht sind.

Die RIDL - Technik soll auch bei anderen Schädlingen, wie dem Baumwollkapselwurm oder der Olivenfliege in absehbarer Zeit eine Zulassung erhalten. Ein entsprechender Antrag der Firma Oxitec für die Zulassung der Olivenfliege in Spanien wurde bereits zweimal, zuletzt im Herbst 2015, zurückgezogen. Auch Freilandversuche wurden bisher von den spanischen Behörden nicht genehmigt. Es wären dies die ersten Freilandversuche mit gentechnisch veränderten Tieren in Europa gewesen.

Der Schädlingsdruck der Olivenfliege, die mit großen Mengen an Insektiziden behandelt wird, ist auf den Olivenplantagen im gesamten Mittelmeerraum groß. Die Fliege legt ihre Eier in bzw. auf die Olivenfrüchte und die Larven ernähren sich dann vom Fruchtfleisch.

Die gentechnisch veränderten Olivenfliegenmännchen tragen ein Genkonstrukt in sich, das dazu führt, dass alle weiblichen Nachkommen bereits im Larvenstadium 
sterben. Der männliche Nachwuchs überlebt, trägt aber wieder das tödliche Gen für alle weiblichen Nachkommen in sich.

Die Menge an Insektiziden, die in den Olivenplantagen eingesetzt wird, ist freilich keinesfalls begrüßenswert. Ob die Ausrottung der Olivenfliege durch gentechnisch veränderte Männchen jedoch einen dauerhaften Erfolg bringen kann, ist fraglich. Die Problematik, dass andere Schädlinge den Platz einnehmen werden, wurde bereits mehrfach angesprochen. Eine Störung des ökologischen Gleichgewichts, die durch das Fehlen einer ganzen Art bestimmt auftritt, kann ungeahnte Folgen nach sich ziehen.

Es ist mit einer Ausbreitung der veränderten Fliege auf den gesamten Mittelmeerraum zu rechnen, weil wohl kein Mechanismus die Olivenfliege bei einem „Freilandversuch“ zu 100 Prozent einsperren kann. Somit kommt ein Testlauf wohl einem Oneway-Ticket gleich.

Die toten Tiere gelangen zudem in die Nahrungskette und werden von Vögeln oder anderen Tieren verspeist. Welche Folgen das haben könnte, ist weitgehend unklar. Lediglich eine Studie, allerdings von Oxitec selbst, sieht darin kein Problem.

Greenpeace argumentiert auch, dass eine Ausbreitung der gentechnisch manipulierten Olivenfliege auf Bio-Olivenplantagen zum Verlust der Bio-Zertifizierung führen und somit einem Zweig der Olivenindustrie vielleicht sogar schaden könnte. Ein vorübergehender Schaden könnte die gesamte Olivenindustrie treffen, wenn nämlich die toten Olivenfliegenlarven nicht aus den Früchten schlüpfen, sondern darin verwesen. Die Oliven würden äußerlich gut aussehen, wären aber für den Verzehr wenig geeignet.

Ein weiteres gentechnisch verändertes Beispielinsekt der Firma Oxitec ist die Kohlmotte. Auch hier soll mit der gleichen Technik wie bei Tigermücke oder Olivenfliege die Art zum Aussterben gebracht werden. Die Vor- und Nachteile sind nahezu ident. Die gentechnisch veränderte Kohlmotte könnte im Bundesstaat New York schon bald in das Freiland entlassen werden. Eine diesbezügliche Entscheidung steht bevor.

\subsection{Säugetiere}

Bei Säugern ist die gentechnische Veränderung etwa mittels Mikroinjektion deutlich komplizierter als etwa bei Fischen. Die Gewinnung der Eizellen ist deutlich mühevoller, sowohl die gentechnische Veränderung mittels Mikroinjektion oder mit Hilfe von Viren als auch das Austragen der Embryonen langwieriger. Um gentechnisch veränderte Merkmale an folgende Generationen weitergeben zu kön- 
nen, ist mitunter auch das Klonen der Tiere notwendig. Dieser gesamte Aufwand bringt zumeist nur extrem niedrige Erfolgsquoten. Die zahlreichen Versuche an Schweinen, die schneller wachsen oder Schafen, die mehr Wolle produzieren sollten, sind nach wie vor immer wieder in den Schlagzeilen, aber letztlich ist in der landwirtschaftlichen Praxis nichts angekommen. Außer der Erfahrung, dass die Tiere, wie eingangs schon angesprochen, während ihres Lebens viele ungünstige Nebeneffekte (vor allem Krankheiten) entwickelten, ist in der Vergangenheit wenig geblieben. In Summe war dieser Bereich wirtschaftlich wenig interessant, weil durch herkömmliche Züchtungsmethoden schon Hochleistungsrassen entstanden sind, so dass das Potential durch gentechnische Veränderung letztlich wohl eher begrenzt war. Trotzdem wird weiter an gentechnisch veränderten Nutztieren gearbeitet und mit den neuen technischen Möglichkeiten wird sich in den nächsten Jahren sehr vieles bewerkstelligen lassen, was bisher nicht möglich oder erfolgreich war. $\mathrm{Ob}$ diese Revolution sich als so vorteilhaft erweisen wird, wie jetzt angepriesen, wird sich zeigen.

Die derzeitigen Anwendungen bei Säugetieren liegen vor allem im Bereich der roten Gentechnik: so gibt es Ziegen, die mit ihrer Milch Medikamente produzieren und unzählige weitere Wirkstoffe aus transgenen Tieren sind in der Pipeline (siehe Kaptitel 7.1). Eine zweite Anwendung liegt in der Transplantation von inneren Organen von Schweinen, die vom menschlichen Immunsystem nicht abgestoßen werden (siehe Kapitel 7.4). Nicht zu vergessen ist auch die wohl wichtigste Anwendung für die moderne Forschung, die unzähligen gentechnisch veränderten Knock-out-Mäuse, die in den Laboren dieser Welt erforscht werden.

Die neue CRISPR/Cas-Methode ist nur eine der neuen Techniken, die noch nicht reguliert und juristisch noch nicht definiert sind und somit keinen speziellen Vorschriften unterliegen. Das führt zu allerlei neuen Meldungen, auch aus dem Bereich der manipulierten Säugetiere. So wurde die DNA von Hunden durch chinesische Forscher so verändert, dass diese mehr Muskelmasse produzieren und somit besser für Jagd- und Polizei-Einsätze geeignet sind. Veränderte Schweine mit doppelt so viel Muskelmasse gegenüber Artgenossen wurden von der Universität in Seoul gemeldet. Auch Resistenzen gegen Krankheiten werden mittels „kleiner“ Modifikationen in den Genen bereits erfolgreich bei Tieren angewendet. Zahlreiche Forschungsprojekte melden Erfolge mit gleichzeitig geringerem Aufwand und niedrigeren Kosten. Praktische Anwendungen und Zulassungsanträge sind nur eine Frage der Zeit. Fraglich ist allerdings, worin genau der Nutzen dieser Möglichkeiten liegt. Noch bedeutender scheint aber eine andere Überlegung: Die Muskeln sind Teil des Bewegungsapparates. Sorgt eine gentechnische Veränderung für eine Zunahme der Muskelmasse, belässt gleichzeitig aber Knochen, Sehnen und das Herz-Kreislauf-System außer acht, so ist fraglich, wie die Tiere mit der 
zusätzlichen Belastung zurechtkommen werden. Normalerweise geht ein Zuwachs an Muskelmasse mit Bewegung, Training und körperlicher Fitness einher und der Körper hat Zeit sich daran anzupassen. Evolutionär entwickelt sich ein Körper als Gesamtes langsam weiter beispielsweise zu einem kräftigeren Körperbau und nicht ein isolierter Teil davon, so wie es durch den gentechnischen Eingriff erfolgt.

Bereits seit den 1980er Jahren gibt es die gentechnische Veränderung an Mäusen. Bei den sogenannten Knockout Mäusen werden durch gezielte gentechnische Eingriffe meist einzelne Gene ausgeschaltet bzw. verändert. Dieser Eingriff erfolgt an embryonalen Mausstammzellen, sodass die Nachkommen ebenfalls diese Eigenschaften tragen. Für die Technik zur Herstellung einer Knockout Maus erhielten drei Forscher 2007 den Nobelpreis für Physiologie oder Medizin. Diese mittlerweile langjährig erprobte Methode wird derzeit durch die neue Genome Editing Methode abgelöst. Mit dieser Technik kann das Mausgenom in befruchteten Maus-Eiern verändert werden ohne embryonale Mausstammzellen. Diese Knockout Maus Produktion in einem Schritt ist demnach weniger aufwendig und somit schneller und billiger.

Mit Hilfe der Knockout Mäuse ist es möglich, einzelne Gene abzuschalten und am lebenden Tier zu erforschen, welche Auswirkungen das hat. So kann man herausfinden, wofür dieses einzelne Gen zuständig ist.

Für manche Fragestellungen werden auch Knockin Mäuse verwendet. Dabei werden keine Gene ausgeschaltet, sondern es wird zusätzliche DNA eingebaut. Der ersten Knockin Maus wurde zum Beispiel ein Krebs auslösendes Gen eingepflanzt, um den Krankheitsverlauf untersuchen zu können.

Der Erfolg der Knockout Mäuse hat in der Praxis auch seine Schattenseiten. In Summe gibt es in den Laboren dieser Welt tausende gentechnisch veränderte Mauslinien, an denen geforscht wird. Mit der Einführung der gentechnischen Veränderung von Versuchstieren ist auch die Zahl der verwendeten Tiere angestiegen. Während die Zahl der Tiere, die zur Prüfung von (giftigen) Substanzen eingesetzt werden leicht zurückgeht, steigt die Anzahl jener Tiere, die im Bereich Grundlagenforschung oder Gentechnik eingesetzt werden. So wurden in Deutschland 2013 über 2 Millionen Mäuse, 375 Tausend Ratten, aber auch 793 Katzen oder 2.542 Hunde verwendet. ${ }^{[4]]}$ In Österreich wurden 2014209.000 Versuchstiere verwendet, wobei auch hier 83 Prozent Mäuse waren. ${ }^{[4]}$ Bei diesen Zahlen sind natürlich nur jene Tiere erfasst, die tatsächlich verwendet wurden, alle anderen scheinen nicht in der Statistik auf. Die „Dunkelziffer“ dürfte aber beeindruckend hoch sein.

Die Tiermodelle sind nach Meinung vieler unverzichtbar. Trotzdem ist etwa in der Grundlagenforschung fraglich, ob alle Fragestellungen eine Verwendung von Versuchstieren rechtfertigen oder auch Alternativen, wie Zelllinien oder Gewebekulturen, ähnliche Ergebnisse bringen könnten. Im Bereich der Medizin ist immer wieder umstritten, ob die Ergebnisse von Mäusen auf den Menschen umgelegt 
werden können, vor allem bei Medikamenten. Ähnliches gilt natürlich auch für die Fütterungsstudien. Trotzdem ist es gängige Praxis, Tiere in unglaublichen Stückzahlen künstlich krank zu machen.

Neben den Mäusen gibt es viele andere Tiermodelle, die zur Simulation von menschlichen Krankheiten, zum Testen von Medikamenten und anderem herangezogen werden. In vielen Laboren der Welt wurden sehr häufig Schimpansen, aufgrund ihrer nahen Verwandtschaft zum Menschen, eingesetzt. Auch deren gentechnische Veränderung ist seit Jahren immer wieder in den Schlagzeilen, vor allem, wenn es um die Diskussion der Patentierung von Lebewesen geht.

Weltweit werden derzeit viele neue Anläufe unternommen um mit neuen genomchirurgischen Eingriffen (z.B.: CRISPR) die DNA der Tiere zu „verbessern“. Auch die Anpassungen der Nutztiere sind dadurch wieder vermehrt in den Fokus gerückt. Schon jetzt häufen sich Meldungen über schnelleres Wachstum, mehr Muskelmasse oder ähnliche „günstige" Eigenschaften, die mit den neuen Verfahren einfacher in die Praxis umzusetzen sind.

Die Politik muss deshalb schnell aktiv werden und sich mit den neuen Methoden der gentechnischen Manipulation auseinandersetzen. Es werden in den nächsten Jahren viele veränderte Tiere marktreif sein und dann braucht es unabhängige Analysen, um Gefahrenpotentiale zu bewerten und auf deren Basis Entscheidungen zu treffen. Die derzeitige Vorgangsweise in vielen Bereichen der Gentechnik, aber auch anderen Bereichen, sich auf Studien der Unternehmen als Bewertungsgrundlage zu verlassen, scheint mehr als zweifelhaft. 